\title{
Antiferromagnetism Emerging in a Ferromagnet with Gain
}

\author{
Huanhuan Yang, C. Wang, Tianlin Yu, Yunshan Cao, ${ }^{*}$ and Peng Yan ${ }^{\dagger}$ \\ School of Electronic Science and Engineering and State Key Laboratory of Electronic Thin Film and Integrated Devices, \\ University of Electronic Science and Technology of China, Chengdu 610054, China
}

(Received 3 July 2018; revised manuscript received 28 August 2018; published 5 November 2018)

\begin{abstract}
We present a theoretical mapping to show that a ferromagnet with gain (loss) is equivalent to an antiferromagnet with an equal amount of loss (gain). Our findings indicate a novel first-order ferromagnetantiferromagnet phase transition by tuning the gain-loss parameter. As an appealing application, we demonstrate the realization as well as the manipulation of the antiferromagnetic Skyrmion, a stable topological quasiparticle not yet observed experimentally, in a chiral ferromagnetic thin film with gain. We also consider ferromagnetic bilayers with balanced gain and loss and show that the antiferromagnetic Skyrmion can be found only in cases with a broken parity-time symmetry phase. Our results pave the way for investigating the emerging antiferromagnetic spintronics and parity-time symmetric magnonics in ferromagnets.
\end{abstract}

DOI: 10.1103/PhysRevLett.121.197201

The first-order antiferromagnetic (AFM) to ferromagnetic (FM) phase transition (or the other way around) has received a tremendous attention in the community of condensed matter physics [1-5]. It involves a transition from a configuration with an antiparallel orientation of the magnetic moments to a parallel configuration, or vice versa. Conventionally, the FM-AFM phase transition is induced by heating [6,7], pressure [8,9], and field $[3,4,10]$, restricted to some specific materials, such as FeRh, $R \mathrm{Mn}_{2} \mathrm{Ge}_{2}$, and Dy, to name a few. It would be very interesting and important if one could find other effective control methods and principles to manipulate the first-order FM-AFM phase transition without the mentioned constraints.

Loss and gain are ubiquitous in nature. Tantalizing physics under their balance has attracted enormous interest and has found many great applications in the context of parity-time $(\mathcal{P} \mathcal{T})$ symmetry and exceptional points (EPs) [11] in a broad field of quantum mechanics [12], optics [13-16], acoustics [17,18], optomechanics [19,20], electronics [21-25], and, very recently, in spintronics [26-30] and cavity spintronics [31,32]. In Ref. [26], Lee et al. proposed two coupled macroscopic FM layers respecting the $\mathcal{P} \mathcal{T}$ symmetry-one layer with loss and another one with an equal amount of gain-and discussed their dynamics in the framework of Landau-Lifshitz-Gilbert (LLG) equation [33]. The positive Gilbert damping (loss) in magnets usually comes from the phonon dissipation and

Published by the American Physical Society under the terms of the Creative Commons Attribution 4.0 International license. Further distribution of this work must maintain attribution to the author(s) and the published article's title, journal citation, and DOI. the electromagnetic radiation, while the negative one (gain) can be realized by parametric driving and/or spin transfer torque $[26,28-30]$. In this Letter, we investigate the properties of microscopic easy-plane "gain" ferromagnets. We map the equation of motion of local magnetic moments to a dissipative one in antiferromagnets and thus argue for their equivalence. Based on this finding, we numerically demonstrate the formation of an AFM Skyrmion, a stable topological quasiparticle yet to be observed experimentally, in single-layer chiral ferromagnets with gain, and we study its dynamics driven by spin-polarized electric currents. We also investigate the spin-wave spectrum in $\mathcal{P} \mathcal{T}$ symmetric bilayer ferromagnets by tuning the balanced gain-loss parameter. The phase diagram of the first-order FM-AFM phase transition is obtained (see Fig. 1). It is interesting that the emerging antiferromagnetism and the AFM Skyrmion can be found only when the $\mathcal{P} \mathcal{T}$ symmetry is broken.

We start with the following Hamiltonian of a ferromagnet in two spatial dimensions (the $x-y$ plane):

$$
\begin{aligned}
\mathcal{H}\left\{\mathbf{m}_{i}\right\}= & -\sum_{\langle i j\rangle} J \mathbf{m}_{i} \cdot \mathbf{m}_{j}-\sum_{\langle i j\rangle} \mathbf{D}_{i j} \cdot\left(\mathbf{m}_{i} \times \mathbf{m}_{j}\right) \\
& +\sum_{i} K\left(\mathbf{m}_{i} \cdot \hat{z}\right)^{2}+\mathcal{H}_{\mathrm{DDI}}\left\{\mathbf{m}_{i}\right\},
\end{aligned}
$$

where $\mathbf{m}_{i}$ is the unit spin vector at the $i$ th site $\left(i_{x} a, i_{y} a\right)$, with $i_{x(y)}$ being an arbitrary integer and $a$ the lattice constant, $J>0$ is the FM exchange coupling constant, $\mathbf{D}_{i j}=D \hat{r}_{i j} \times \hat{z}$ is the interfacial Dzyaloshinskii-Moriya interaction (DMI) vector [34,35] with the unit vector $\hat{r}_{i j}=$ $\mathbf{r}_{i j} / r_{i j}$ connecting sites $i$ and $j$ at a distance $r_{i j}=\left|\mathbf{r}_{i j}\right|$, $\langle i j\rangle$ sums over all nearest-neighbor sites, $K>0$ is the easy-plane magnetic anisotropy constant, and 

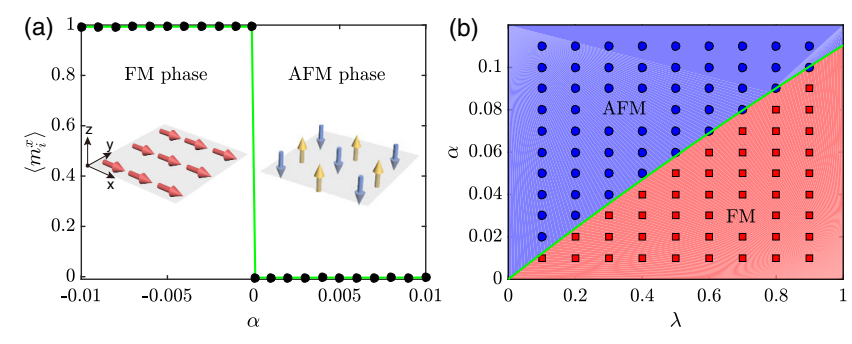

FIG. 1. (a) Order parameter $\left\langle m_{i}^{x}\right\rangle$ as a function of $\alpha$ in a single FM layer. The dots are numerical results. (Inset) Spin configurations of FM and AFM states. (b) Phase diagram of the $\mathcal{P} \mathcal{T}$ symmetric bilayer by tuning the gain-loss parameter $\alpha$ and the interlayer coupling constant $\lambda$. Symbols are numerical results, and the green curve is the analytical formula $\min _{\forall \mathbf{k}} \alpha_{c}(\mathbf{k})=$ $\lambda /\left[2 \sqrt{\zeta_{0}\left(\lambda+\zeta_{0}\right)}\right]$.

$$
\mathcal{H}_{\mathrm{DDI}}\left\{\mathbf{m}_{i}\right\}=\sum_{i \neq j} \frac{\mu_{0} M_{s}^{2} a^{6}}{4 \pi r_{i j}^{3}}\left[\mathbf{m}_{i} \cdot \mathbf{m}_{j}-3\left(\mathbf{m}_{i} \cdot \hat{r}_{i j}\right)\left(\mathbf{m}_{j} \cdot \hat{r}_{i j}\right)\right]
$$

is the nonlocal dipolar interaction, with $\mu_{0}$ being the vacuum magnetic permeability and $M_{s}$ the saturation magnetization. Ideal magnetic materials for the energy model (1) are $\mathrm{Fe}_{0.7} \mathrm{Co}_{0.3} \mathrm{Si}$ [36], $\mathrm{CoFeB}$ [37], etc. The generalization of the present model to generic twodimensional (2D) magnets with, e.g., honeycomb lattices, next-nearest-neighbor interactions, or different anisotropy axes, is straightforward.

In a ferromagnet with gain, the time evolution of the magnetization dynamics can be described by the modified LLG equation $[26,33]$

$$
\frac{d \mathbf{m}_{i}}{d t}=-\gamma \mathbf{m}_{i} \times \mathbf{H}_{\mathrm{eff}, i}-\alpha \mathbf{m}_{i} \times \frac{d \mathbf{m}_{i}}{d t},
$$

where $\gamma$ is the (positive) gyromagnetic ratio and $\alpha>0$ is the gain coefficient. The first term in the right-hand side of Eq. (3) describes the Larmor precession of local spins about the effective field $\mathbf{H}_{\text {eff }, i}=-\left(\mu_{0} M_{s} a^{3}\right)^{-1} \partial \mathcal{H}\left\{\mathbf{m}_{i}\right\} / \partial \mathbf{m}_{i}$. The second term is a torque driving the spin away from the field. Owing to the very presence of the gain, the energy change rate of the spin system

$$
\frac{d \mathcal{H}}{d t}=\frac{\alpha \gamma \mu_{0} M_{s} a^{3}}{1+\alpha^{2}} \sum_{i}\left|\mathbf{m}_{i} \times \mathbf{H}_{\mathrm{eff}, i}\right|^{2}
$$

is always non-negative. The parallel state of magnetizations in the ferromagnet is thus unstable, and the system seeks the energy maximum.

To obtain more insights, we utilize a mapping $\mathbf{n}_{i}=-\mathbf{m}_{i}$ and recast Eq. (3) into

$$
\frac{d \mathbf{n}_{i}}{d t}=-\gamma \mathbf{n}_{i} \times \tilde{\mathbf{H}}_{\mathrm{eff}, i}+\alpha \mathbf{n}_{i} \times \frac{d \mathbf{n}_{i}}{d t},
$$

which recovers the dissipative LLG equation describing the collective motion of spin vectors $\mathbf{n}_{i}$ governed by a new Hamiltonian $\tilde{\mathcal{H}}\left\{\mathbf{n}_{i}\right\}=-\mathcal{H}\left\{\mathbf{n}_{i}\right\}$, with $\tilde{\mathbf{H}}_{\text {eff }, i}=$ $-\left(\mu_{0} M_{s} a^{3}\right)^{-1} \partial \tilde{\mathcal{H}}\left\{\mathbf{n}_{i}\right\} / \partial \mathbf{n}_{i}$. Interestingly, we note that $\tilde{\mathcal{H}}$ can be interpreted as a 2D AFM Hamiltonian, with $J$ being the AFM exchange constant and $K$ the easy-axis anisotropy constant along the $z$ direction. While the physical meaning of a negative dipolar interaction $-\mathcal{H}_{\mathrm{DDI}}\left\{\mathbf{n}_{i}\right\}$ is not so transparent, we find that the dipole-dipole interaction effectively renormalizes the exchange constant as $J \rightarrow J-$ $\mu_{0} M_{s}^{2} a^{3} /(4 \pi)$ when the stabilized magnetizations are aligned in an antiparallel manner by expanding the Hamiltonian for nearest neighbors. This correction, however, usually is negligibly small: In $\mathrm{Fe}_{0.7} \mathrm{Co}_{0.3} \mathrm{Si}$ [36], for instance, the ratio $\mu_{0} M_{s}^{2} a^{3} /(4 \pi J) \sim 10^{-4}$. We thus conclude that a ferromagnet with gain is equivalent to an antiferromagnet with an equal amount of loss. The statement can be presented the other way around as well: An antiferromagnet with gain is equivalent to a ferromagnet with the same loss.

Then we show that the above mapping indicates a firstorder FM-AFM phase transition by tuning the gain-loss parameter $\alpha$. To this end, we choose the magnetization $\left\langle m_{i}^{x}\right\rangle$ as the order parameter, with $\langle\cdots\rangle$ representing the average over all sites, and numerically solve Eq. (3) with the MuMAX3 package [38]. We use materials parameters of $\mathrm{Fe}_{0.7} \mathrm{Co}_{0.3} \mathrm{Si}$ [39]. By systematically changing the parameter $\alpha$, we observe a sharp transition of the order parameter at the point $\alpha=0$, as shown in Fig. 1(a). The internal magnetization configuration changes from a FM state to an AFM one, as depicted in the inset. This is direct evidence of the first-order FM-AFM phase transition. Further, we find that for bilayer ferromagnets with the $\mathcal{P} \mathcal{T}$ symmetry, the first-order phase transition point coincides with the EP, depending on both the gain-loss parameter and the interlayer coupling constant, as shown in Fig. 1(b) (see the derivations and discussions below).

Now we introduce one compelling application of our findings on Skyrmion generations and manipulations. It is common wisdom that Skyrmions cannot stabilize in a single easy-plane ferromagnet without applying the external magnetic field perpendicular to the plane $[36,40,41]$. We challenge this view by realizing an AFM Skyrmion in a FM thin film with gain. In the simulations, we choose a fixed gain parameter $\alpha=0.01$.

We start with a random initial $(t=0)$ magnetization profile [see Fig. 2(a)], which mimics the state of the thermal demagnetization, for instance. At $t=0.035 \mathrm{ns,} \mathrm{local}$ magnetic moments quickly evolve to an antiparallelly aligned state, as shown in Fig. 2(b). We therefore achieve an AFM state in a ferromagnet with the energy cost $2 \times 60^{2} J \approx 9.4 \mathrm{eV}$. The average power is estimated to be as low as $43 \mathrm{nW}$, comparable to that used to excite spin waves in FM thin films [42]. However, the Skyrmionic spin texture is yet to emerge. In Fig. 2(c), we randomize all spins inside a circle of radius $5 \mathrm{~nm}$ in the film center, which can 


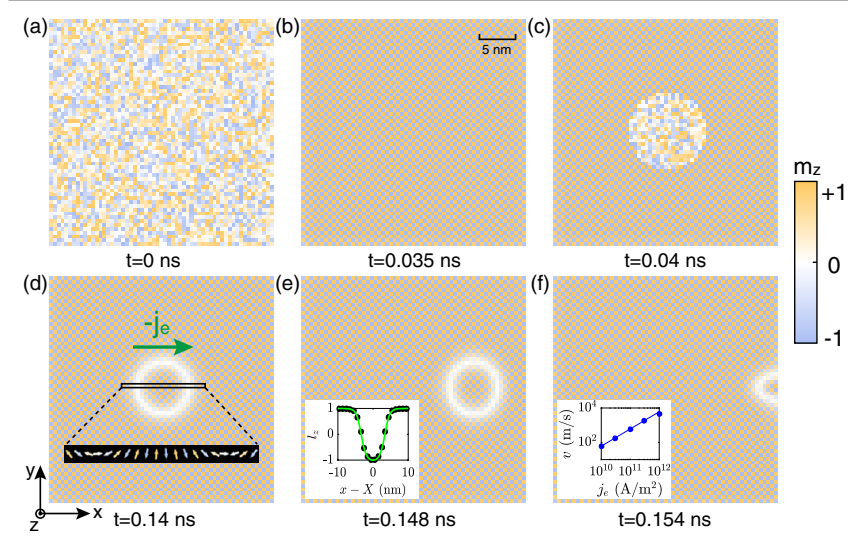

FIG. 2. (a) Random spin configuration at $t=0$. (b) AFM state evolved at $t=0.035 \mathrm{~ns}$. (c) Randomizing spins inside the circle at $t=0.04 \mathrm{~ns}$. (d) AFM Skyrmion stabilized at $t=0.14 \mathrm{~ns}$. (Inset) Magnetization profile of the cross section of the AFM Skyrmion. (e) Current-driven AFM Skyrmion motion. (Inset) Spatial distribution of the $z$ component of the Néel vector $\mathbf{l}_{i}$, with $X$ being the position of the Skyrmion center. The dots are numerical results and the green curve is a fitting with Eq. (7) in Ref. [44]. (f) AFM Skyrmion annihilation at the film boundary. (Inset) Current dependence of the Skyrmion velocity when it is far from the edge. The dots are numerical results, and the solid line is the analytical formula.

be realized by local heatings or current pulses [43]. At $t=0.14 \mathrm{~ns}$, an AFM Skyrmion stabilizes [see Fig. 2(d)]. The profile of the staggered magnetization of the AFM Skyrmion can be well described by the formula proposed in Ref. [44], as shown in the inset of Fig. 2(e).

To manipulate the AFM Skyrmion motion, we apply an in-plane spin-polarized electric current $\mathbf{j}_{e}=-j_{e} \hat{x}$ with $j_{e}=5.0 \times 10^{11} \mathrm{~A} \mathrm{~m}^{-2}$. We find that the AFM Skyrmion propagates with a large velocity $3000 \mathrm{~m} \mathrm{~s}^{-1}$. We note that the Skyrmion trajectory is exactly along the flowing direction of electrons, without suffering the Skyrmion Hall effect [see Fig. 2(e)]. The high speed of the AFM Skyrmion agrees with the formula $\mathbf{v}=(\beta / \alpha) u \hat{x}$ obtained from Thiele's equation $[45,46]$, as shown in the inset of Fig. 2(f), where $u=\mu_{B} j_{e} /\left[|e| M_{s}\left(1+\beta^{2}\right)\right]$ is the drift velocity of conduction electrons, with $\mu_{B}$ being the Bohr magneton, $e$ the electron charge, and $\beta$ the material nonadiabatic parameter (we set $\beta=0.1$ in the simulations). We do not observe any visible difference with or without dipolar fields. Owing to the repulsive force from the boundary, the AFM Skyrmion is slowed down but finally annihilates at the edge since the driving force from the current overcomes the edge repulsion, as shown in Fig. 2(f). All these features of the AFM Skyrmion motion can be well reproduced by simulating (5) instead of (3) (not shown).

Compared to their FM counterparts, AFM Skyrmions [47] have some other advantages, such as the elevated mobility [48-51] and unusual thermal properties [48,52], among others [53-57]. One recent breakthrough toward (a)
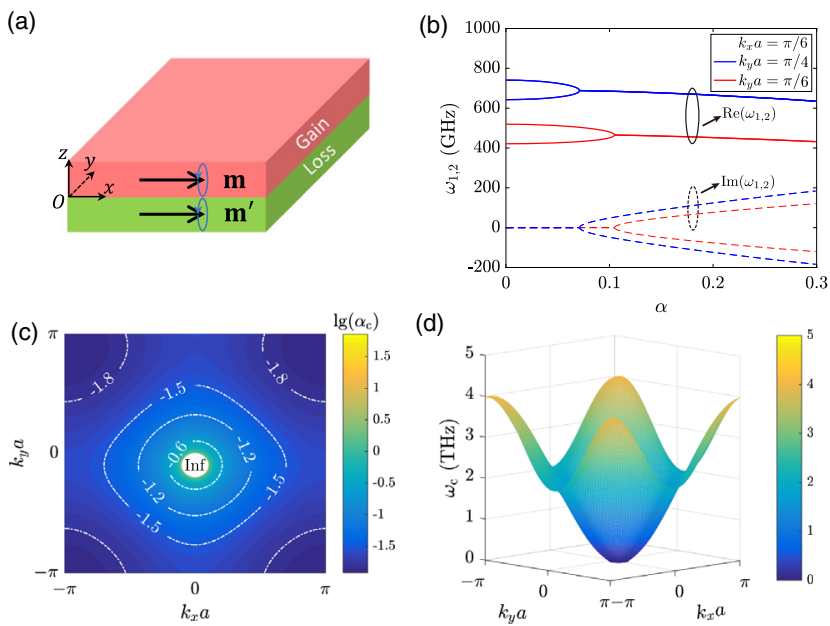

FIG. 3. (a) Schematic plot of coupled FM bilayers $\left(30 \times 30 \times 1 \mathrm{~nm}^{3}\right)$ with balanced gain (red layer) and loss (green layer) with equilibrium magnetizations along the $\hat{x}$ direction. (b) Evolution of $\omega_{1,2}$ on $\alpha$ for two representative spin-wave modes $\mathbf{k}=\left[\left(\frac{\pi}{6 a}\right),\left(\frac{\pi}{4 a}\right)\right]$ (blue curves) and $\left[\left(\frac{\pi}{6 a}\right),\left(\frac{\pi}{6 a}\right)\right]$ (red curves). (c) Contour plot of the mode dependence of $\alpha_{c}$. $\mathcal{P} \mathcal{T}$ symmetry is never broken in the white region labeled "Inf", the abbreviation for infinity. (d) Critical frequency $\omega_{c}$ as a function of $\mathbf{k}$. In the calculations, we adopted the materials parameters of $\mathrm{Fe}_{0.7} \mathrm{Co}_{0.3} \mathrm{Si}$ and the interlayer coupling constant $\lambda=0.1$.

this direction is the experimental realization of ferrimagnetic Skyrmions in GdFeCo films with an inhibited Skyrmion Hall effect [58,59]. Because of its intrinsic difficulties in materials and detections, the AFM Skyrmion is yet to be observed in experiments. Our strategy to generate the AFM Skyrmion in single-layer ferromagnets thus provides a possible way to overcome the barrier.

We next extend the original idea of Ref. [26] to two coupled FM films by including finite intralayer exchange couplings, which enables us to investigate the spin-wave (magnon) excitations. The schematic setup is shown in Fig. 3(a), with $\mathbf{m}$ and $\mathbf{m}^{\prime}$ representing the spatiotemporal magnetization direction in the layer with gain and the layer with loss, respectively. The equations of motion for the coupled magnetization dynamics read

$$
\begin{aligned}
\frac{d \mathbf{m}_{i}}{d t} & =-\gamma \mathbf{m}_{i} \times\left[\mathbf{H}_{\mathrm{eff}, i}+\lambda\left(J / \mu_{0} M_{s} a^{3}\right) \mathbf{m}_{i}^{\prime}\right]-\alpha \mathbf{m}_{i} \times \frac{d \mathbf{m}_{i}}{d t}, \\
\frac{d \mathbf{m}_{i}^{\prime}}{d t} & =-\gamma \mathbf{m}_{i}^{\prime} \times\left[\mathbf{H}_{\mathrm{eff}, i}^{\prime}+\lambda\left(J / \mu_{0} M_{s} a^{3}\right) \mathbf{m}_{i}\right]+\alpha \mathbf{m}_{i}^{\prime} \times \frac{d \mathbf{m}_{i}^{\prime}}{d t},
\end{aligned}
$$

where $\mathbf{H}_{\mathrm{eff}, i}^{\prime}$ is made identical to $\mathbf{H}_{\mathrm{eff}, i}$ in Eq. (3) by replacing its constituent $\mathbf{m}$ with $\mathbf{m}^{\prime}$, and $\lambda>0$ is the ratio between the interlayer and the intralayer exchange coupling. Under a combined operation of parity $\mathcal{P}\left(\mathbf{m}_{i} \leftrightarrow \mathbf{m}_{i}^{\prime}\right.$ and $\left.\mathbf{H}_{\mathrm{eff}, i} \leftrightarrow \mathbf{H}_{\mathrm{eff}, i}^{\prime}\right)$ and time reversal $\mathcal{T}\left(t \rightarrow-t, \mathbf{m}_{i} \rightarrow\right.$ $-\mathbf{m}_{i}, \mathbf{m}_{i}^{\prime} \rightarrow-\mathbf{m}_{i}^{\prime}, \mathbf{H}_{\mathrm{eff}, i} \rightarrow-\mathbf{H}_{\mathrm{eff}, i}$, and $\left.\mathbf{H}_{\mathrm{eff}, i}^{\prime} \rightarrow-\mathbf{H}_{\mathrm{eff}, i}^{\prime}\right)$, 
we find that Eqs. (6) are invariant and thus respect the $\mathcal{P} \mathcal{T}$ symmetry. To obtain the spin-wave spectrum, we consider a small deviation of both $\mathbf{m}_{i}$ and $\mathbf{m}_{i}^{\prime}$ from their equilibrium direction $\hat{x}: \mathbf{m}_{i}=\left(1, \delta m_{i, y}, \delta m_{i, z}\right)$ and $\mathbf{m}_{i}^{\prime}=\left(1, \delta m_{i, y}^{\prime}, \delta m_{i, z}^{\prime}\right)$, with $\left|\delta m_{i, y}\right|+\left|\delta m_{i, z}\right|+\left|\delta m_{i, y}^{\prime}\right|+\left|\delta m_{i, z}^{\prime}\right| \ll 1$. The eigensolutions of linearized equations (6) have the forms of $\delta m_{i, y}=Y e^{i(\mathbf{k} \cdot \mathbf{r}-\omega t)}, \quad \delta m_{i, z}=Z e^{i(\mathbf{k} \cdot \mathbf{r}-\omega t)} \quad$ and $\quad \delta m_{i, y}^{\prime}=$ $Y^{\prime} e^{i(\mathbf{k} \cdot \mathbf{r}-\omega t)}, \delta m_{i, z}^{\prime}=Z^{\prime} e^{i(\mathbf{k} \cdot \mathbf{r}-\omega t)}$, with $\mathbf{r}=\left(i_{x}, i_{y}\right) a$ and $\mathbf{k}=\left(k_{x}, k_{y}\right)$ being the wave vector of the spin wave. We thus obtain the equation for the column vector $\Psi(\mathbf{k})=$ $\left(Y, Z, Y^{\prime}, Z^{\prime}\right)^{\mathrm{T}}$ :

$$
H(\mathbf{k}) \Psi(\mathbf{k})=\omega(\mathbf{k}) \Psi(\mathbf{k})
$$

where $H$ is a $4 \times 4$ matrix

$$
H(\mathbf{k})=\frac{\gamma}{\left(1+\alpha^{2}\right) \mu_{0} M_{s} a^{3}}\left(\begin{array}{cccc}
\chi_{1}(\mathbf{k})+\alpha\left[\chi_{2}^{*}(\mathbf{k})-2 i K^{\prime}\right] & \chi_{2}(\mathbf{k})+\alpha \chi_{1}(\mathbf{k}) & \alpha \chi_{0}^{*} & \chi_{0} \\
-\alpha \chi_{1}(\mathbf{k})+\chi_{2}^{*}(\mathbf{k})-2 i K^{\prime} & -\alpha \chi_{2}(\mathbf{k})+\chi_{1}(\mathbf{k}) & \chi_{0}^{*} & \alpha \chi_{0}^{*} \\
\alpha \chi_{0} & \chi_{0} & \chi_{1}(\mathbf{k})-\alpha\left[\chi_{2}^{*}(\mathbf{k})-2 i K^{\prime}\right] & \chi_{2}(\mathbf{k})-\alpha \chi_{1}(\mathbf{k}) \\
\chi_{0}^{*} & \alpha \chi_{0} & \alpha \chi_{1}(\mathbf{k})+\chi_{2}^{*}(\mathbf{k})-2 i K^{\prime} & \alpha \chi_{2}(\mathbf{k})+\chi_{1}(\mathbf{k})
\end{array}\right) \text {, }
$$

with $\chi_{0}=i \lambda J, \chi_{1}(\mathbf{k})=2 D \sin k_{y} a, \chi_{2}(\mathbf{k})=2 i J\left(\cos k_{x} a+\right.$ $\left.\cos k_{y} a\right)-i(4+\lambda) J-2 i K^{\prime}$, and $K^{\prime}=K+\mu_{0} M_{s}^{2} a^{3} / 2$ summing up the easy-plane anisotropy and the demagnetizing energy. The solutions of eigenfrequencies come in pairs $\pm \omega$. Two positive solutions, corresponding to counterclockwise magnetization precession around the ground state along the $\hat{x}$ direction, are relevant and can be expressed as

$$
\omega_{1,2}(\mathbf{k})=\lambda+2 \zeta(\mathbf{k}) \pm \sqrt{\lambda^{2}-4 \alpha^{2} \zeta(\mathbf{k})[\lambda+\zeta(\mathbf{k})]}
$$

multiplied by $\gamma J /\left[\left(1+\alpha^{2}\right) \mu_{0} M_{s} a^{3}\right]$, with $\zeta(\mathbf{k})=2-$ $\cos k_{x} a-\cos k_{y} a+(D / J) \sin k_{y} a$. In deriving Eq. (9), we have dropped the contribution from $K^{\prime}$ since we focus on the exchange spin-wave region. For a given $\mathbf{k}$, as the gain and loss parameter $\alpha$ increases, the two eigenfrequencies approach one another, and at some critical value $\alpha=\alpha_{c}$ they coalesce at the $\mathrm{EP}$ and bifurcate into the complex plane [see Fig. 3(b)]. At the EP, the two normal modes coalesce as well. The domain with real eigenfrequencies is termed the exact phase, otherwise known as the broken phase. From Eq. (9), one can obtain both the critical gain-loss parameter and the critical frequency. Nevertheless, we point out a special region $-\lambda \leq \zeta(\mathbf{k}) \leq 0$ in which the $\mathcal{P} \mathcal{T}$ symmetry is never broken without considering the nonlinear effect of the LLG equations (6). This fact is in contrast to conventional $\mathcal{P} \mathcal{T}$ symmetric systems suffering symmetry breaking when the strength of the gainloss term exceeds a certain critical value [26]. Of course, the nonlinear magnon-magnon interaction complicates this picture and will generate a level broadening of spin-wave eigenmodes [42]. For $\zeta(\mathbf{k})$ outside $[-\lambda, 0]$, the two critical parameters are given by

$$
\begin{aligned}
& \alpha_{c}(\mathbf{k})=\frac{\lambda}{2 \sqrt{\zeta(\mathbf{k})[\lambda+\zeta(\mathbf{k})]}} \\
& \omega_{c}(\mathbf{k})=\frac{\gamma J}{\mu_{0} M_{s} a^{3}} \frac{\lambda+2 \zeta(\mathbf{k})}{1+\alpha_{c}^{2}(\mathbf{k})}
\end{aligned}
$$

both of which are mode dependent. Figures 3(c) and 3(d) show the distribution of $\alpha_{c}$ and $\omega_{c}$ over the first Brillouin zone, respectively. The center of the white region in Fig. 3(c) does not coincide with the origin, with a downward shift $\arctan (D / J)$ caused by the DMI.

In the exact phase $\alpha<\min _{\forall \mathbf{k}} \alpha_{c}(\mathbf{k})=\lambda /\left[2 \sqrt{\zeta_{0}\left(\lambda+\zeta_{0}\right)}\right]$ with $\zeta_{0}=3+\sqrt{1+(D / J)^{2}}$, predictions from the linear spin-wave theory compare well with the full simulation of Eqs. (6) that the steady-state magnetizations in both layers oscillate around the initial misalignment from the $\hat{x}$ axis without being attenuated or amplified (see Fig. 4). Since both layers are in the FM state in the exact phase, we observe only the counterclockwise spin-wave modes.

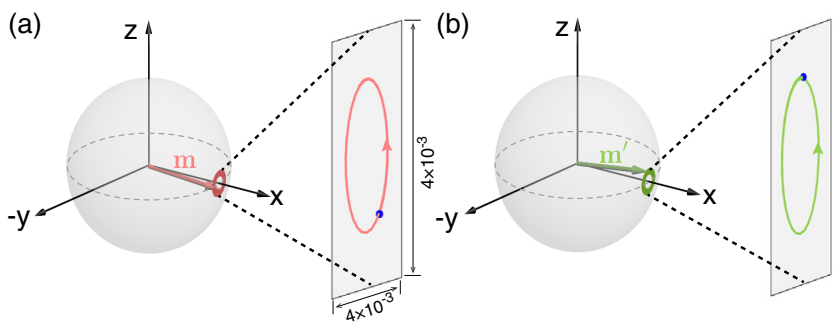

FIG. 4. Trajectory of the steady-state magnetizations at the site $(30 a, 30 a)$ in both (a) the gain layer and (b) the lossy layer in the exact phase, with enlarged details shown on the right side. The blue dot indicates the instantaneous phase of the spin. We set pinned boundary conditions and $\alpha=0.01$ with the same rest parameters as were used in Fig. 3. 


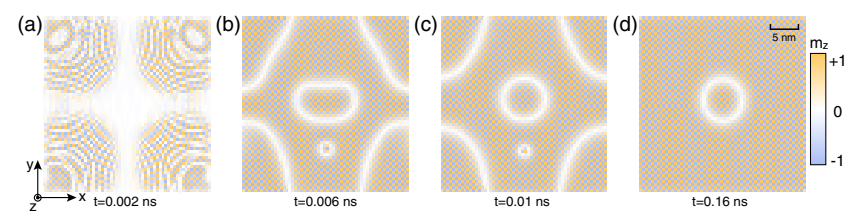

FIG. 5. Time evolution of magnetizations in the gain layer under the broken phase, at (a) $t=0.002 \mathrm{ns,} \mathrm{(b)} t=0.006 \mathrm{ns,}$ (c) $t=0.01 \mathrm{~ns}$, and (d) $t=0.16 \mathrm{~ns} . \alpha=0.3$ in the simulation.

In the broken phase $\alpha>\lambda /\left[2 \sqrt{\zeta_{0}\left(\lambda+\zeta_{0}\right)}\right] \approx 0.012$ for $\lambda=0.1$, the linear theory indicates an exponential growth of the spin-wave amplitude, which is associated with the case in which the eigenfrequencies (9) have an imaginary part. The induced instability can drive the spin away from its equilibrium direction. The situation in the critical phase $\alpha=\lambda /\left[2 \sqrt{\zeta_{0}\left(\lambda+\zeta_{0}\right)}\right]$ is similar: The linear spin-wave theory introduces a linear instead of an exponential growth of the wave amplitude, which is the consequence of the EP degeneracy. The lossy layer thus preserves the in-plane FM state to some extent (not shown). However, in the gain layer, it is interesting that the original in-plane magnetizations along the $\hat{x}$ direction evolve to be perpendicular to the plane (but with a negligibly small global canting of an angle less than $2.5^{\circ}$ with respect to the normal of the lossy layer) and finally form an AFM Skyrmion, as shown in Figs. 5(a)-5(d). The EP thus exactly coincides with the FM-AFM phase transition point in the gain layer, which has been verified by micromagnetic simulations; see Fig. 1(b).

Negative damping is essential to realizing our proposal. Its real world implementation methods are multiform, besides the two approaches introduced above. A recent experiment reported the electric-field-induced negative magnetic damping in FM|FE (ferroelectric) heterostructures [60]. In Ref. [61], Wegrowe et al. thoroughly analyzed the spin transfer in an open FM layer, and they found that the negative damping appears naturally for describing the exchange of spins between the magnetic system and the environment [62-64].

In summary, we uncovered a mapping between a ferromagnet with gain and an antiferromagnet with an equal amount of loss. A novel first-order FM to AFM phase transition, or vice versa, was predicted by tuning the gainloss parameter. In a chiral easy-plane ferromagnet in the presence of gain, we showed the emergence of a stabilized AFM Skyrmion without applying any external field. In 1D and 2D nonchiral gain ferromagnets, we envision the formation of AFM domain walls [65] and vortices [66], respectively. We also studied the spin-wave spectrum in FM bilayers with balanced gain and loss. We predicted a spectral region in the first Brillouin zone, in which the $\mathcal{P} \mathcal{T}$ symmetry is never broken in the framework of linear spinwave theory. We found that the emerging antiferromagnetism and the AFM Skyrmion appear in the gain layer only in cases of a broken $\mathcal{P} \mathcal{T}$ symmetry phase. The results presented here open a new way to create and manipulate AFM solitons in simple ferromagnets through the firstorder FM-AFM phase transition, and they build a novel bridge connecting the $\mathcal{P} \mathcal{T}$ symmetry to magnonics and Skyrmionics.

This work is funded by the National Natural Science Foundation of China (Grants No. 11704060 and No. 11604041), the National Key Research Development Program under Contract No. 2016YFA0300801, and the National Thousand-Young-Talent Program of China. C. W. acknowledges financial support from the China Postdoctoral Science Foundation (Grants No. 2017M610595 and No. 2017T100684) and the National Natural Science Foundation of China under Grant No. 11704061.

H. Y. and C. W. contributed equally to this work.

*yunshan.cao@uestc.edu.cn †yan@uestc.edu.cn

[1] C. Kittel, Model of exchange-inversion magnetization, Phys. Rev. 120, 335 (1960).

[2] A. Zakharov, Crystal lattice parameter and structural distortions in Fe-Rh alloy at phase transitions, Fiz. Met. Metalloved. 24, 84 (1967).

[3] J. H. V. J. Brabers, K. H. J. Buschow, and F. R. de Boer, Field-induced first-order antiferromagnetic-ferromagnetic transitions in $R \mathrm{Mn}_{2} \mathrm{Ge}_{2}$ compounds and their relation to the magnetostriction of the Mn sublattice, Phys. Rev. B 59, 9314 (1999).

[4] X. Marti et al., Room-temperature antiferromagnetic memory resistor, Nat. Mater. 13, 367 (2014).

[5] C. Gatel, B. Warot-Fonrose, N. Biziere, L. A. Rodríguez, D. Reyes, R. Cours, M. Castiella, and M. J. Casanove, Inhomogeneous spatial distribution of the magnetic transition in an iron-rhodium thin film, Nat. Commun. 8, 15703 (2017).

[6] C. Stamm, J.-U. Thiele, T. Kachel, I. Radu, P. Ramm, M. Kosuth, J. Minár, H. Ebert, H. A. Dürr, W. Eberhardt, and C. H. Back, Antiferromagnetic-ferromagnetic phase transition in FeRh probed by x-ray magnetic circular dichroism, Phys. Rev. B 77, 184401 (2008).

[7] A. Chirkova, F. Bittner, K. Nenkov, N. V. Baranov, L. Schultz, K. Nielsch, and T. G. Woodcock, The effect of the microstructure on the antiferromagnetic to ferromagnetic transition in FeRh alloys, Acta Mater. 131, 31 (2017).

[8] J. A. Ricodeau and D. Melville, Model of the antiferromagnetic-ferromagnetic transition in FeRh alloys, J. Phys. F 2, 337 (1972).

[9] S. Li, Z. Ao, J. Zhu, J. Ren, J. Yi, G. Wang, and W. Liu, Strain controlled ferromagnetic-antiferromagnetic transformation in Mn-doped silicene for information transformation devices, J. Phys. Chem. Lett. 8, 1484 (2017).

[10] Y. Lee, Z. Q. Liu, J. T. Heron, J. D. Clarkson, J. Hong, C. Ko, M. D. Biegalski, U. Aschauer, S. L. Hsu, M. E. Nowakowski, J. Wu, H. M. Christen, S. Salahuddin, J. B. Bokor, N. A. Spaldin, D. G. Schlom, and R. Ramesh, Large resistivity modulation in mixed-phase metallic systems, Nat. Commun. 6, 5959 (2015). 
[11] V. V. Konotop, J. Yang, and D. A. Zezyulin, Nonlinear waves in $\mathcal{P} \mathcal{T}$-symmetric systems, Rev. Mod. Phys. 88, 035002 (2016).

[12] C. M. Bender and S. Boettcher, Real Spectra in NonHermitian Hamiltonians having $\mathcal{P} \mathcal{T}$ Symmetry, Phys. Rev. Lett. 80, 5243 (1998).

[13] K. G. Makris, R. El-Ganainy, D. N. Christodoulides, and Z. H. Musslimani, Beam Dynamics in $\mathcal{P} \mathcal{T}$ Symmetric Optical Lattices, Phys. Rev. Lett. 100, 103904 (2008).

[14] C. E. Rüter, K. G. Makris, R. El-Ganainy, D. N. Christodoulides, M. Segev, and D. Kip, Observation of parity-time symmetry in optics, Nat. Phys. 6, 192 (2010).

[15] A. Regensburger, C. Bersch, M.-A. Miri, G. Onishchukov, D. N. Christodoulides, and U. Peschel, Parity-time synthetic photonic lattices, Nature (London) 488, 167 (2012).

[16] X.-L. Zhang, S. Wang, B. Hou, and C. T. Chan, Dynamically Encircling Exceptional Points: In situ Control of Encircling Loops and the Role of the Starting Point, Phys. Rev. X 8, 021066 (2018).

[17] X. Zhu, H. Ramezani, C. Shi, J. Zhu, and X. Zhang, $\mathcal{P} \mathcal{T}$ Symmetric Acoustics, Phys. Rev. X 4, 031042 (2014).

[18] R. Fleury, D. Sounas, and A. Alù, An invisible acoustic sensor based on parity-time symmetry, Nat. Commun. 6, 5905 (2015).

[19] X. Y. Lu, H. Jing, J. Y. Ma, and Y. Wu, PT -SymmetryBreaking Chaos in Optomechanics, Phys. Rev. Lett. 114, 253601 (2015).

[20] H. Xu, D. Mason, L. Jiang, and J. G. E. Harris, Topological energy transfer in an optomechanical system with exceptional points, Nature (London) 537, 80 (2016).

[21] J. Schindler, A. Li, M. C. Zheng, F. M. Ellis, and T. Kottos, Experimental study of active LRC circuits with $\mathcal{P} \mathcal{T}$ symmetries, Phys. Rev. A 84, 040101(R) (2011).

[22] J. Schindler, Z. Lin, J. M. Lee, H. Ramezani, F. M. Ellis, and T. Kottos, $\mathcal{P} \mathcal{T}$-symmetric electronics, J. Phys. A 45, 444029 (2012).

[23] N. Bender, S. Factor, J. D. Bodyfelt, H. Ramezani, D. N. Christodoulides, F. M. Ellis, and T. Kottos, Observation of Asymmetric Transport in Structures with Active Nonlinearities, Phys. Rev. Lett. 110, 234101 (2013).

[24] S. Assawaworrarit, X. Yu, and S. Fan, Robust wireless power transfer using a nonlinear parity-time-symmetric circuit, Nature (London) 546, 387 (2017).

[25] P.-Y. Chen, M. Sakhdari, M. Hajizadegan, Q. Cui, M. M.-C. Cheng, R. El-Ganainy, and A. Alù, Generalized parity-time symmetry condition for enhanced sensor telemetry, Nat. Electron. 1, 297 (2018).

[26] J. M. Lee, T. Kottos, and B. Shapiro, Macroscopic magnetic structures with balanced gain and loss, Phys. Rev. B 91, 094416 (2015).

[27] P. Yan, J. Sinova, and G. E. W. Bauer, Dynamics of Ferromagnetic Bilayers, poster presented at Gordon Research Conference (GRC) "Spin Dynamics in Nanostructures," Kowloon, Hong Kong, 2015.

[28] A. Galda and V. M. Vinokur, Parity-time symmetry breaking in magnetic systems, Phys. Rev. B 94, 020408(R) (2016).

[29] A. Galda and V. M. Vinokur, Linear dynamics of classical spin as Möbius transformation, Sci. Rep. 7, 1168 (2017).

[30] A. Galda and V. M. Vinokur, Parity-time symmetry breaking in spin chains, Phys. Rev. B 97, 201411(R) (2018).
[31] M. Harder, L. Bai, P. Hyde, and C.-M. Hu, Topological properties of a coupled spin-photon system induced by damping, Phys. Rev. B 95, 214411 (2017).

[32] D. Zhang, X.-Q. Luo, Y.-P. Wang, T.-F. Li, and J. Q. You, Observation of the exceptional point in cavity magnonpolaritons, Nat. Commun. 8, 1368 (2017).

[33] T. L. Gilbert, A phenomenological theory of damping in ferromagnetic materials, IEEE Trans. Magn. 40, 3443 (2004).

[34] I. Dzyaloshinsky, A thermodynamic theory of "weak" ferromagnetism of antiferromagnetics, J. Phys. Chem. Solids 4, 241 (1958).

[35] T. Moriya, Anisotropic superexchange interaction and weak ferromagnetism, Phys. Rev. 120, 91 (1960).

[36] M. Vousden, M. Albert, M. Beg, M.-A. Bisotti, R. Carey, D. Chernyshenko, D. Cortés-Ortuño, W. Wang, O. Hovorka, C. H. Marrows, and H. Fangohr, Skyrmions in thin films with easy-plane magnetocrystalline anisotropy, Appl. Phys. Lett. 108, 132406 (2016).

[37] J.-W. Xu, V. Sluka, B. Kardasz, M. Pinarbasi, and A. D. Kent, Ferromagnetic resonance linewidth in coupled layers with easy-plane and perpendicular magnetic anisotropies, J. Appl. Phys. 124, 063902 (2018).

[38] A. Vansteenkiste, J. Leliaert, M. Dvornik, M. Helsen, F. Garcia-Sanchez, and B. Van Waeyenberge, The design and verification of MuMAX3, AIP Adv. 4, 107133 (2014).

[39] The materials parameters of $\mathrm{Fe}_{0.7} \mathrm{Co}_{0.3} \mathrm{Si}$ are [36] the saturation magnetization $M_{s}=9.5 \times 10^{4} \mathrm{~A} / \mathrm{m}$, the lattice constant $a=0.5 \mathrm{~nm}$, the ferromagnetic exchange constant $J=1.3 \mathrm{meV}$, the DMI strength $D=0.42 \mathrm{meV}$, and the magnetocrystalline anisotropy coefficient $K=0.12 \mathrm{meV}$. We consider a sample of size $30 \times 30 \times 0.5 \mathrm{~nm}^{3}$ with the free boundary condition.

[40] A. O. Leonov and I. Kézsmárki, Asymmetric isolated skyrmions in polar magnets with easy-plane anisotropy, Phys. Rev. B 96, 014423 (2017).

[41] S.-Z. Lin, A. Saxena, and C. D. Batista, Skyrmion fractionalization and merons in chiral magnets with easy-plane anisotropy, Phys. Rev. B 91, 224407 (2015).

[42] B. Zhang, Z. Wang, Y. Cao, P. Yan, and X. R. Wang, Eavesdropping on spin waves inside the domain-wall nanochannel via three-magnon processes, Phys. Rev. B 97, 094421 (2018).

[43] H. Y. Yuan and X. R. Wang, Skyrmion creation and manipulation by nano-second current pulses, Sci. Rep. 6, 22638 (2016).

[44] X. S. Wang, H. Y. Yuan, and X. R. Wang, A theory on skyrmion size, Commun. Phys. 1, 31 (2018).

[45] A. A. Thiele, Steady-State Motion of Magnetic Domains, Phys. Rev. Lett. 30, 230 (1973).

[46] H. Yang, C. Wang, X. Wang, X. S. Wang, Y. Cao, and P. Yan, Twisted skyrmions at domain boundaries and the method of image skyrmions, Phys. Rev. B 98, 014433 (2018).

[47] A. N. Bogdanov and D. A. Yablonskii, Contribution to the theory of inhomogeneous states of magnets in the region of magnetic-field-induced phase transitions. Mixed state of antiferromagnets, Zh. Eksp. Teor. Fiz. 96, 253 (1989).

[48] J. Barker and O. A. Tretiakov, Static and Dynamical Properties of Antiferromagnetic Skyrmions in the Presence of Applied Current and Temperature, Phys. Rev. Lett. 116, 147203 (2016). 
[49] X. Zhang, Y. Zhou, and M. Ezawa, Antiferromagnetic skyrmion: Stability, creation and manipulation, Sci. Rep. 6, 24795 (2016).

[50] C. Jin, C. Song, J. Wang, and Q. Liu, Dynamics of antiferromagnetic skyrmion driven by the spin Hall effect, Appl. Phys. Lett. 109, 182404 (2016).

[51] H. Velkov, O. Gomonay, M. Beens, G. Schwiete, A. Brataas, J. Sinova, and R. A. Duine, Phenomenology of currentinduced skyrmion motion in antiferromagnets, New J. Phys. 18, 075016 (2016).

[52] W. Yang, H. Yang, Y. Cao, and P. Yan, Photonic orbital angular momentum transfer and magnetic skyrmion rotation, Opt. Express 26, 8778 (2018).

[53] R. Keesman, M. Raaijmakers, A. E. Baerends, G. T. Barkema, and R. A. Duine, Skyrmions in square-lattice antiferromagnets, Phys. Rev. B 94, 054402 (2016).

[54] P. M. Buhl, F. Freimuth, S. Blügel, and Y. Mokrousov, Topological spin Hall effect in antiferromagnetic skyrmions, Phys. Status Solidi RRL 11, 1700007 (2017).

[55] B. Göbel, A. Mook, J. Henk, and I. Mertig, Antiferromagnetic skyrmion crystals: Generation, topological Hall, and topological spin Hall effect, Phys. Rev. B 96, 060406(R) (2017).

[56] C.-C. Liu, P. Goswami, and Q. Si, Skyrmion defects and competing singlet orders in a half-filled antiferromagnetic Kondo-Heisenberg model on the honeycomb lattice, Phys. Rev. B 96, 125101 (2017).

[57] X. Zhao, R. Ren, G. Xie, and Y. Liu, Single antiferromagnetic skyrmion transistor based on strain manipulation, Appl. Phys. Lett. 112, 252402 (2018).

[58] S. Woo, K. M. Song, X. C. Zhang, Y. Zhou, M. Ezawa, X. Liu, S. Finizio, J. Raabe, N. J. Lee, S.-II Kim, S.-Y. Park, Y. Kim, J.-Y. Kim, D. Lee, O. Lee, J. W. Choi, B.-C. Min, H. C. Koo, and J. Chang, Current-driven dynamics and inhibition of the skyrmion Hall effect of ferrimagnetic skyrmions in GdFeCo films, Nat. Commun. 9, 959 (2018).
[59] S. Woo, K. M. Song, X. C. Zhang, M. Ezawa, Y. Zhou, X. Liu, M. Weigand, S. Finizio, J. Raabe, M.-C. Park, K.-Y. Lee, J. W. Choi, B.-C. Min, H. C. Koo, and J. Chang, Deterministic creation and deletion of a single magnetic skyrmion observed by direct time-resolved X-ray microscopy, Nat. Electron. 1, 288 (2018).

[60] C. L. Jia, F. L. Wang, C. J. Jiang, J. Berakdar, and D. S. Xue, Electric tuning of magnetization dynamics and electric field-induced negative magnetic permeability in nanoscale composite multiferroics, Sci. Rep. 5, 11111 (2015).

[61] J.-E. Wegrowe, M. C. Ciornei, and H.-J. Drouhin, Spin transfer in an open ferromagnetic layer: From negative damping to effective temperature, J. Phys. Condens. Matter 19, 165213 (2007).

[62] Z. Li and S. Zhang, Magnetization dynamics with a spintransfer torque, Phys. Rev. B 68, 024404 (2003).

[63] K. Ando, S. Takahashi, K. Harii, K. Sasage, J. Ieda, S. Maekawa, and E. Saitoh, Electric Manipulation of Spin Relaxation Using the Spin Hall Effect, Phys. Rev. Lett. 101, 036601 (2008).

[64] Z. Duan, A. Smith, L. Yang, B. Youngblood, J. Lindner, V. E. Demidov, S. O. Demokritov, and I. N. Krivorotov, Nanowire spin torque oscillator driven by spin orbit torques, Nat. Commun. 5, 5616 (2014).

[65] J. Li, A. Tan, S. Ma, R. F. Yang, E. Arenholz, C. Hwang, and Z. Q. Qiu, Chirality Switching and Winding or Unwinding of the Antiferromagnetic $\mathrm{NiO}$ Domain Walls in $\mathrm{Fe} / \mathrm{NiO} / \mathrm{Fe} / \mathrm{CoO} / \mathrm{Ag}(001)$, Phys. Rev. Lett. 113, 147207 (2014).

[66] J. Wu, D. Carlton, J. S. Park, Y. Meng, E. Arenholz, A. Doran, A. T. Young, A. Scholl, C. Hwang, H. W. Zhao, J. Bokor, and Z. Q. Qiu, Direct observation of imprinted antiferromagnetic vortex states in $\mathrm{CoO} / \mathrm{Fe} / \mathrm{Ag}(001)$ discs, Nat. Phys. 7, 303 (2011). 